International Journal of Wireless \& Mobile Networks (IJWMN) Vol. 5, No. 6, December 2013

\title{
AREVIEW OF SECURITY ATtACKS AND INTRUSION DETECTION SCHEMES IN WIRELESS SENSOR NETWORK
}

\author{
Yassine MALEH ${ }^{1}$ and Abdellah Ezzati ${ }^{2}$ \\ Emerging Technologies Laboratory (VETE), Faculty of Sciences and Technology \\ Hassan $1{ }^{\text {st }}$ University, Settat, MOROCCO
}

\begin{abstract}
Wireless sensor networks are currently the greatest innovation in the field of telecommunications. WSNs have a wide range of potential applications, including security and surveillance, control, actuation and maintenance of complex systems and fine-grain monitoring of indoor and outdoor environments. However security is one of the major aspects of Wireless sensor networks due to the resource limitations of sensor nodes. Those networks are facing several threats that affect their functioning and their life. In this paper we present security attacks in wireless sensor networks, and we focus on comparison and analysis of recent Intrusion Detection schemes in WSNs.
\end{abstract}

\section{KEYWORDS}

Wireless sensor Networks, Security, attack, Denial of Service (DoS), Intrusion Detection Systems (IDS), IDS Architectures, Cluster-based IDS, Anomaly-based IDS, Signature based IDS\&Hybrid IDS

\section{INTRODUCTION}

Recent advances in wireless and micro electronic communications have enabled the development of a new type of wireless network called wireless sensor network (WSN).Wireless sensor networks are associated with vulnerable characteristics such as open-air transmission and selforganizing without a fixed infrastructure [1]. Consequently security of wireless sensor networks (WSN) is the most challenge for this type of network [2]. Intrusion Detection Systems (IDSs) can play an important role in detecting and preventing security attacks. This paper presents a review of the security attacks in wireless sensor network and analyzed some of the existing IDS models and architectures. Finally a comparative study and a discussion of IDS models will be presented.

\section{RELATED WORK}

Wireless sensor networks are not immune to the risks of destruction and decommissioning. Some of these risks are identical to those in Ad-Hoc networks, and others are specific to the sensors. Several articles [6][7][8][9][10] have presented security attacks and issues in WSNs. Intrusion detection system (IDS) defined as the second line of defense after cryptography, allows the detection and prevention of internal and external attacks.

In [18, it is presented a Rule-based IDS called also Signature-based. Most of the techniques in these schemes follow three main phases: data acquisition phase, rule application phase and

DOI : $10.5121 /$ ijwmn.2013.5606 
intrusion detection phase.In [19], it is proposed two approaches to improve the security of clusters for sensor networks using IDS. The first approach usesamodel-based on authentication, and the second scheme is called Energy-Saving.IN [21] a hybrid intrusion detection system (HIDS) model has been anticipated for wireless sensor networks.This paper does not promote a solution. Rather, it is a comparative study of existing model of intrusion detection in wireless sensor networks. Our aim is to provide a better understanding of the current research issues in this field.

\section{Security GoALS IN WSN}

We can classify the security goals into two goals:mainand secondary. The main goals include security objectives that should be available in any system (confidentiality, availability, integrityandauthentication). The other category includes secondary goals (self-organization, secure localization, Time synchronization andResilience to attacks) [3] [4].

- Confidentiality (Forbid access to unwanted third parties)

- Authentication (Identity verification and validation)

- Availability (Service has to be always available)

- Integrity (Data is exchanged without malicious alteration)

- Self Organization(Every sensor node needs to be independent and flexible enough to be selforganizing and self-healing)

- Secure localization (Sensor network often needs location information accurately and automatically)

- Time synchronization (Sensor radio may be turned off periodically in order to conserve power)

- Resilience to attacks (The covenant of a single node must not violate the security of the whole network).Figure1belowsummarizessecurity goals for wirelesssensor network.

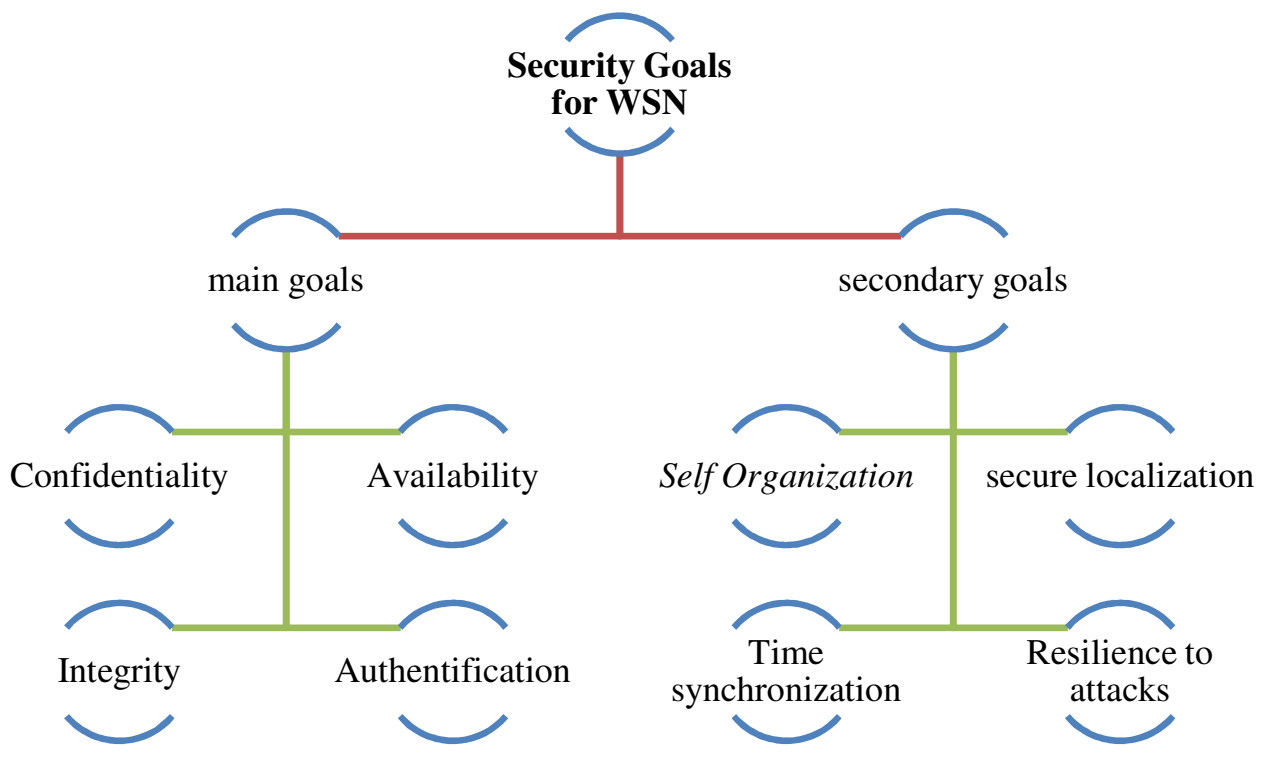

Figure1.Security Goals for WSN 
International Journal of Wireless \& Mobile Networks (IJWMN) Vol. 5, No. 6, December 2013

\section{SECURITY ATtaCKS IN WSN}

The different characteristics of wireless sensor networks (energy limited, low-power computing, use of radio waves, etc...) expose them to many securitythreats. We can classify the attacks into two main categories [5]: Active and Passive. In passive attacks, attackers are typically camouflaged, i.e. hidden, and tap the communication lines to collect data. In active attacks, malicious acts are carried out not only against data confidentiality but also data integrity.Several papers havepresentedthesecurityattacks in WSN [6][7][8][9][10].

\section{- Spoofed, altered or replayed routing information}

May be used for loop construction, attracting or repelling traffic, extend or shorten source route.

\section{- Selectiveforwarding}

In this attack, the attacker prevents the transmission of some packets. They will be removed later by the malicious node.

\section{- Worm holeattack:}

Thewormholeattackrequiresinsertion atleasttwomaliciousnodes. Thesetwonodesareinterconnectedbyapowerful connection for examplea wired link. The malicious node receives packets in one section of the network and sends them to another section of the network.

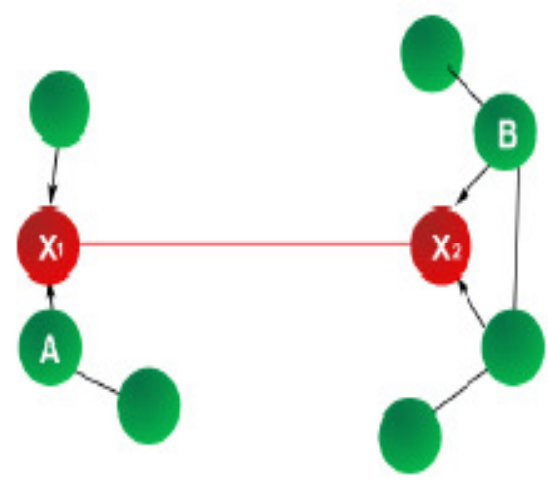

Figure 2. Worm hole attack

\section{- Sybil attack:}

A malicious node presents multiple identities to the other nodes in the network. This poses a significant threat to routing protocols and will cause the saturation of the routing tables of the nodes with incorrect information. 


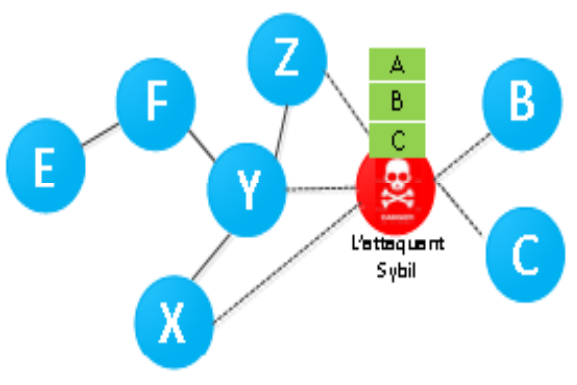

Figure 3. Sybil attack

\section{- Black hole attack:}

The attackinvolves inserting amaliciousnode in the network. This node, by various means, will modify the routing tablesto force themaximumneighboringnodespassingtheinformationthroughhim.Thenlike a black holeinspace,allthe information that willgoinitwill never beretransmitted.

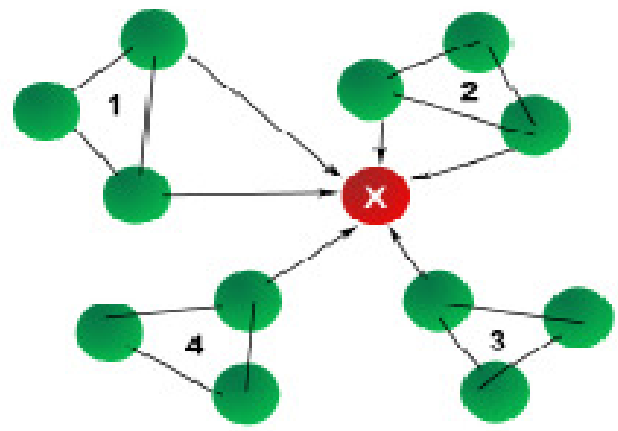

Figure 4. Black hole attack

\section{- Hello Flooding:}

Discoveryprotocolson WSNs useHELLOmessagestypesto discover itsneighboring nodes. In an attacktypeHELLOFlooding, an attacker will usethismechanismto saturate thenetworkandconsumeenergy.

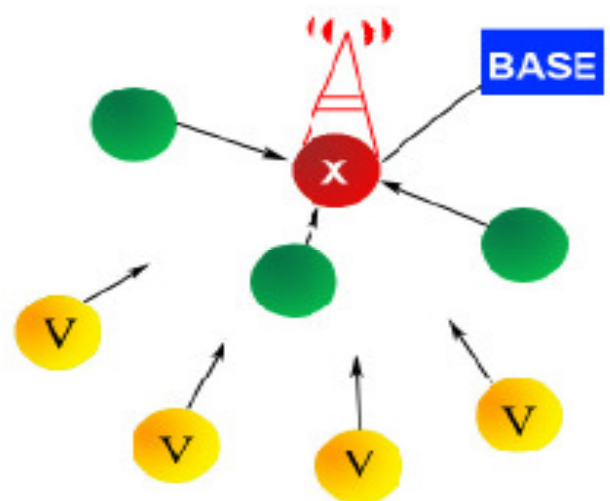

Figure 5. Hello flooding attack 


\section{- Acknowledgementspoofing}

In this attack, the attacker tries to convince the sender that the weak link is strong or that a dead node is alive. Therefore, all packets passing through this link or this node will be lost.

\section{- Denial-of-Service Attacks}

A denial-of-service (DoS) targets the availability and capacity reduction of network services. Physical constraints of the sensor networks and the nature of their deployment environment, make them vulnerable to DoS attacks more than any other type of network. In this section we will review important DoS scenarios for each layer of the WSN. In [11] Wang et al. (2006) have classified the DoS attacks that could target each layer of the WSN.

\begin{tabular}{|c|c|c|}
\hline Layer & Attacks & Defense \\
\hline Physical & Jamming & $\begin{array}{l}\text { Spread-spectrum, priority messages, } \\
\text { lower duty cycle, region mapping, } \\
\text { mode change }\end{array}$ \\
\hline \multirow{3}{*}{ Link } & Collision & Error-correction code \\
\hline & Exhaustion & Rate limitation \\
\hline & Unfairness & Small frames \\
\hline \multirow{5}{*}{ Network } & $\begin{array}{l}\text { Spoofed routing } \\
\text { information, andselective } \\
\text { forwarding }\end{array}$ & $\begin{array}{l}\text { Egressfiltering, } \\
\text { authentication, monitoring }\end{array}$ \\
\hline & Sinkhole & Redundancychecking \\
\hline & Sybil & $\begin{array}{l}\text { Authentication, } \\
\text { monitoring, redundancy }\end{array}$ \\
\hline & Wormhole & Authentication, probing \\
\hline & Hello Flood & Authentication \\
\hline \multirow{2}{*}{ Transport } & Session Hijacking. & aggregationdata \\
\hline & SYN flooding & Package authentication \\
\hline Application & $\begin{array}{l}\text { Data Corruption. } \\
\text { Repudiation }\end{array}$ & Authentification \\
\hline
\end{tabular}

Table 1. Various DOS attacks on WSNs and their countermeasures 


\section{COUNTERMEASURES}

To counter the attacks threatened networks wireless sensors, several research teams are trying to find appropriate solutions. These solutions must take into account the specificities of wireless sensor networks. We need to find simple solutions to secure the network while consuming the least possible energy and adapt these solutions to a low power computing. In the range of these solutions include mechanisms such as data partitioning, the use of appropriate cryptographic methods, intruder detection by location or even the confidence index. Wood and Stankovic [12] studied DoS attacks and possible defense. In [13][14]a suite of optimized security protocols for wireless sensor network is presented. SPIN (Security Protocol for Information via Negotiation) has two security mechanisms: SNEP and TESLA. SNEP provides data confidentiality and data authentication. TESLA provides source authentication in multicast scenarios by using MAC chaining. It is based on loose time synchronization between the sender and the receivers. INSENS (Intrusion Tolerant routing for wireless sensor networks) this protocol allows the base station to draw an accurate map of the network that will establish the routing tables for each node [15]. Du,et al. [16] propose LEAP+ (Localized Encryption and Authentication Protocol), a key management protocol for sensor networks.

\section{INTRUSION DETECTIONSYSTEMSINWSN}

Afterthe concept ofintrusiondetection(ID), which was established in 1980,two major variants of intrusion detection systems (IDS) have emerged, Host intrusion detection systems (HIDS) and network intrusion detection systems (NIDS) [17]. Intrusion detection is an approach that is complementary with respect to mainstream of security mechanisms such as cryptography and access control [18]. Intrusion detectioncan be defined as Intrusion detectioncan be defined astheautomatic detection andalarmgenerationtoreportthatan intrusion hasoccurredoris in progress. Inthis section we describethe architecture ofIDSinWSNs. IDS cannot takepreventive action, since they arepassivein nature, they can only detect intrusion and generate an alarm. The following figure presents the four main components of IDS [19].

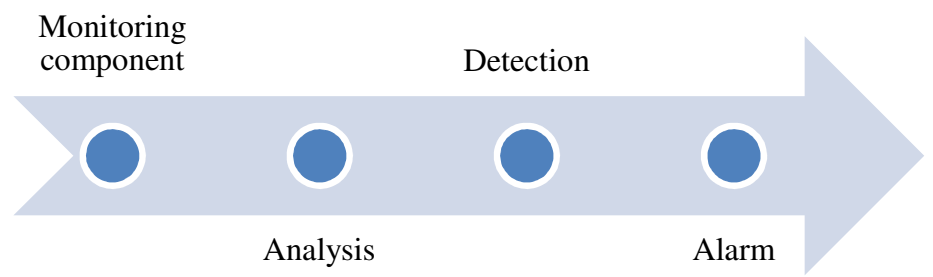

Figure 6.IDS components

There are two distinct technologies of IDS:

- Network Intrusion Detection System (NIDS). These systems are designed to intercept and analyze packets circulating in the network. All communication in the wireless network are 
conducted on the air and a node can hear the traffic passing from a neighboring node (promiscuous mode) [36]. Therefore, the nodes can mutually check the network traffic. This technology applies this concept, IDS listens for traffic and individually examine each packet.

- Host intrusion detection systems (HIDS). Analysis only data on the node where the IDS is installed. Any decision is based on information collected at this node. These IDSs use two types of sources to provide information about the activity: the log files (file that records all activity on a system in standby), and audit trails ( Incoming / outgoing packets node, etc).

\subsection{The challenging of designing IDS for WSN}

The IDS solutions developed for wired networks cannot be applied directly to sensor networks, view the difference between these two types of networks, this is why it is necessary to introduce an intrusion detection system that meets the special features of sensor networks[20]. The design of this kind of system for wireless sensor network must satisfy the following properties:

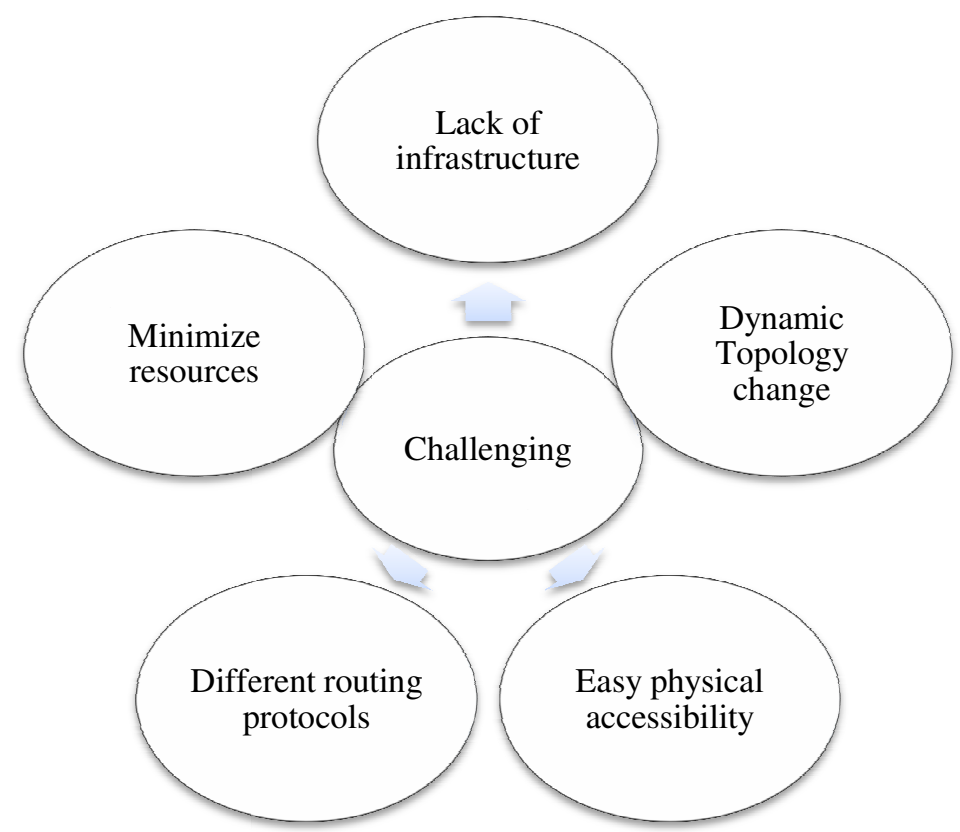

Figure 7. Challenging of designing IDS for WSN

\subsection{The requirements of designing IDS for WSN}

In wireless sensor networks, the IDS must satisfy the following properties [21]:

- Localize auditing: IDS forwirelesssensor networks mustworkwithlocaldataandpartialaudits, because in WSN there arenocentralizedpoints(apart from thestationbase) thatcancollectglobaldataauditing.

- Minimize resources: IDS must use a minimum number of resources for networks.Communicationbetweennodesforintrusiondetectionshouldnotsaturatetheavailable bandwidth.

- Trust no node: Unlikewired networks, nodes sensors canbecompromisedeasily, IDS must nottrustanynode.

- Bedistributed: means that thecollectionand analysis ofdata should beinseveral locations. Moreoverthedistributedapproachalsoappliestotheexecution of the algorithmofdetectionandalert correlation.

- Besecure: IDSmust be able towithstand attacks. 
Figure 8 below summarizes requirements of designing IDS for WSN.

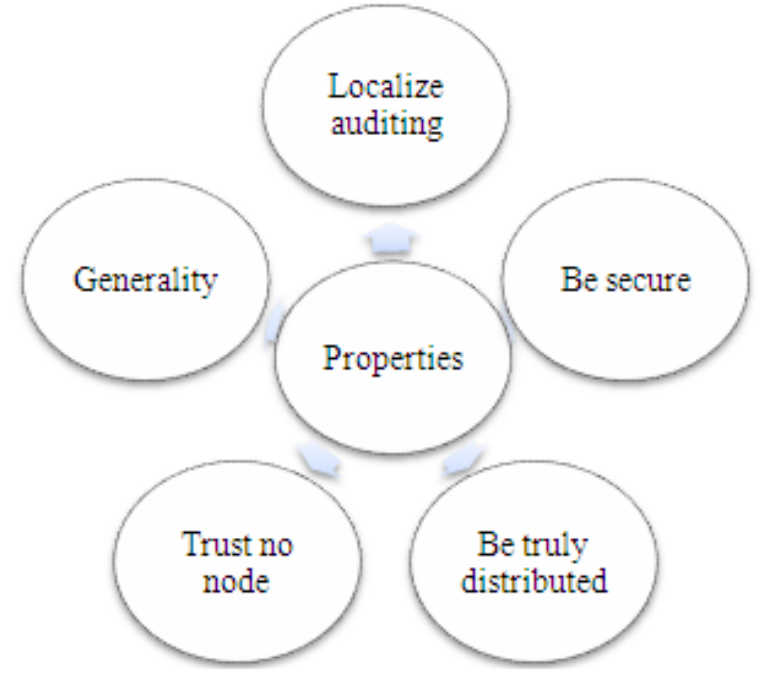

Figure 8.Requirements of designing IDS for WSN

\subsection{Architectures for IDS in Wireless Sensor Network}

The nature of wireless sensor networks makes them very vulnerable to attack.The Mobile nodes are randomly distributed, there are no physical obstacles for the adversary, therefore, they can be easily captured, and attacks can come from all directions and target any node. To tackle these additional challenges, several possible IDS architectures exist including standalone IDS, distributed and cooperative IDS and hierarchical IDS [22].

\subsubsection{Standalone IDS}

In this category, each node operatesas independent IDSandisresponsibleforthe detection ofattacksagainsthim.Therefore, theIDSdo not cooperateanddo not shareinformation with each other.This architecturerequires that eachnodeiscapable of executing and running IDS.

\subsubsection{Distributed and Cooperative IDS}

In this architecture (Zhang et al., 2003), each node has an IDS agent and makes local detection decisions by itself, all the nodes cooperate to create a global detection process. The distributed and cooperative IDS architecture is more suitable for a flat network configuration than a clusterbased multilayered one.

\subsubsection{Hierarchical IDS}

In this category the network is divided into clusters with cluster-heads. In each cluster, a leader plays the role of cluster-head. This node is responsible for routing in the group and must accept messages from members of the cluster indicating something malicious. Similarly, the cluster-head must detect attacks against other cluster-heads in the network. At the same time all cluster-heads can cooperate with central base station to form global IDS. 


\subsection{Some open research in IDS}

Cross-Layer IDS: Using a cross layer IDS, we could not only pass information between layers but also coordinate mechanisms to prevent threats at all layers.

Dynamic IDS: The IDS that would protect mobile nodes, as in VANET networks.

Internet of Things IDS: There should be mechanisms that could manage all the objects of our everyday life that have an IP address and be connected to the Internet.

\section{INTRUSION DETECTION MODELS FOR WSN}

Due toarchitecturaldifferencebetweenwiredandwireless networks, their IDSs cannot be used interchangeably. There are specific techniques for WSN [23]. In this section, we analyze and discus some proposed IDSs for WSN.

\subsection{Rule-based IDS}

Rule-based IDS called also Signature-based IDS, articulates on a database of stored prior rules of security attacks [24]. Most of the techniques in these schemes follow three main phases: data acquisition phase, rule application phase and intrusion detection phase (Silva et al., 2005) [25].The algorithmincludesthreestepsfor detecting intrusions. In the first step monitor nodes monitors the data. In the second step detection ruleswillberankedin order of severity, to the collected information to flag failure. The third step is the intrusion detection phase, where the number of failure flagged is compared to the expected number of the occasional failures in the network.

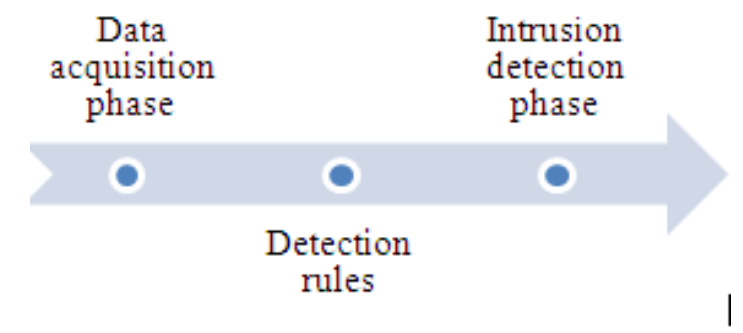

Figure 9.Steps for detecting intrusion in rule based IDS

\subsection{Cluster-Based IDS}

$\mathrm{Su}$, et al. [26] hasproposed two approaches to improve the security of clusters for sensor networks using IDS. The first approach usesamodel-based on authentication, which can resisttoexternalattacks. Its basic technique is to add a message authentication code (MAC) for each message. Whenever a node wants to send a message, it adds to it a timestamp and a MAC is generated by a key-pair or individually depending on the key role of the sender (cluster-head, member -node, or base station). So that the receiver can verify the sender, the security mechanism is used LEAP. The second scheme is called Energy-Saving. This approach focuses on the detection of misbehavior both in Member nodes (MN) and cluster-head nodes $(\mathrm{CH})$. When misbehavior is detected, the $\mathrm{CH}$ broadcasts a warning message encrypted with the cluster key to restrain this specific node.

\subsection{Hybrid IDS}

In the Hybrid Approach, both techniques (Cluster-Based and Rule-Based) arecombinedto form Hybrid detection technique.Hybrid detection exploitsthe advantages of bothapproachesprovides 
simplicity, high safety, low consumption of energy [27] [28].The Hybrid Intrusion Detection System achieves the goals of high detection rate and low false positive rate.

\section{ANALYSES AND DISCUSSION}

Comparing analysis, for the advantages and drawbacks of different models:

Rule-Based : The rule based model is simple, clear levels, anddesigned for a large-sized WSNs. Signature-based IDS need more resource than anomaly-based IDS, and regular updatingofthe database with new attack signatures.

Cluster-based: The cluster-based model requires Low Energy Consumption, provideshigh level of security. Because of Centralized routing data delivery is guaranteed. In cluster-based IDS Message retransmission frequency is high, and the centralized routing may not always use best available path for routing.

Hybrid model: Hybrid model are designed for large and sustainable WSN. Thismodelusestwomechanisms, anomaly-based and signature-based, so it requireshighconsumption of energy.

Table 2 gives the comparison and characteristics of different IDSs.

\begin{tabular}{|c|c|c|c|c|c|c|}
\hline $\begin{array}{l}\text { IDS } \\
\text { Model }\end{array}$ & $\begin{array}{l}\text { Network } \\
\text { architecture }\end{array}$ & $\begin{array}{l}\text { Detection } \\
\text { technique }\end{array}$ & $\begin{array}{l}\text { Handled } \\
\text { attacks }\end{array}$ & $\begin{array}{l}\text { Energy } \\
\text { consumption }\end{array}$ & Advantages & Drawbacks \\
\hline $\begin{array}{l}\text { Anomaly } \\
\text { based IDS }\end{array}$ & & $\begin{array}{l}\text { Anomaly } \\
\text { Based }\end{array}$ & $\begin{array}{l}\text { Masquera } \\
\text { de, } \\
\text { routing } \\
\text { attacks, } \\
\text { Sinkhole } \\
\text { and } \\
\text { blackhole }\end{array}$ & Low & $\begin{array}{l}\text { Capable of } \\
\text { detecting } \\
\text { new attacks }\end{array}$ & $\begin{array}{l}\text { Misses } \\
\text { wellknown } \\
\text { attack }\end{array}$ \\
\hline $\begin{array}{l}\text { Rule- } \\
\text { based IDS }\end{array}$ & Distributed & $\begin{array}{l}\text { Signatur } \\
\text { e } \\
\text { based }\end{array}$ & $\begin{array}{l}\text { Black } \\
\text { hole, } \\
\text { selective } \\
\text { forwardin } \\
\text { g, Sink } \\
\text { hole, } \\
\text { DOS }\end{array}$ & Low & $\begin{array}{l}\text { Detects all } \\
\text { those } \\
\text { attacks } \\
\text { having } \\
\text { signatures }\end{array}$ & $\begin{array}{l}\text { Cannotdetect } \\
\text { new } \\
\text { attacks }\end{array}$ \\
\hline $\begin{array}{l}\text { Cluster- } \\
\text { based IDS }\end{array}$ & Hierarchical & $\begin{array}{l}\text { Anomaly } \\
\text { Based }\end{array}$ & & Low & $\begin{array}{l}\text { Low } \\
\text { Energy } \\
\text { Consumpti } \\
\text { on } \\
\text { Data del } \\
\text { ivery is } \\
\text { guaranteed }\end{array}$ & $\begin{array}{l}\text { Message } \\
\text { retransmissio } \\
\mathrm{n} \text { frequency is } \\
\text { high, } \\
\text { Increased } \\
\text { Traffic }\end{array}$ \\
\hline $\begin{array}{l}\text { Hybrid } \\
\text { IDS }\end{array}$ & Hierarchical & $\begin{array}{l}\text { Anomaly } \\
\text { based }\end{array}$ & $\begin{array}{l}\text { Selective } \\
\text { forwardin } \\
\mathrm{g}, \\
\text { sinkhole, } \\
\text { Hello } \\
\text { flood and } \\
\text { wormhole } \\
\text { attacks }\end{array}$ & Medium & $\begin{array}{l}\text { Can detect } \\
\text { both } \\
\text { existing } \\
\text { and new } \\
\text { attacks }\end{array}$ & $\begin{array}{l}\text { Requires } \\
\text { more } \\
\text { computation } \\
\text { and } \\
\text { resources }\end{array}$ \\
\hline
\end{tabular}


International Journal of Wireless \& Mobile Networks (IJWMN) Vol. 5, No. 6, December 2013

\begin{tabular}{|l|l|l|l|l|l|l|}
\hline $\begin{array}{l}\text { Intrusion } \\
\text { detection } \\
\text { in Routing } \\
\text { Attacks }\end{array}$ & Distributed & $\begin{array}{l}\text { Anomaly } \\
\text { based }\end{array}$ & $\begin{array}{l}\text { DoS, } \\
\text { Sinkhole } \\
\text { and } \\
\text { wormhole } \\
\text { attacks }\end{array}$ & High & $\begin{array}{l}\text { Consider } \\
\text { resource } \\
\text { Parameters }\end{array}$ & $\begin{array}{l}\text { High resource } \\
\text { Requirement, } \\
\text { Increased } \\
\text { (energy } \\
\text { and } \\
\text { reliability) }\end{array}$ \\
\hline
\end{tabular}

\section{CONCLUSION}

This article shows how well a security sensor networks is a challenge for researchers and developers of information technology.Our goal was to present the existing security attacks in WSN, focusing on intrusion detection systems (IDS), and examine existing approaches of intrusion detection in WSN.Our goal was to present the existing security mechanisms for WSN, specifically focusing on intrusion detection systems (IDS), and consider existing approaches to provide a fairly comprehensive and effective model. We are now working on our own model that incorporates all the advantages of the approaches proposed for a global model of intrusion detection in WSN.

\section{REFERENCES}

[1] Gang Zhao, "Wireless Sensor Networks for Industrial Process Monitoring and Control: A Survey", Network Protocols and Algorithms, ISSN 1943-358, Vol. 3, No. 1, 2011.

[2] G. Padmavath, D. Shanmugapriya, "A Survey of Attacks, Security Mechanisms and Challenges in Wireless Sensor Networks”, (IJCSIS) International Journal of Computer Science and Information Security, Vol. 4, No. 1 \& 2, 2009.

[3] QusayIdreesSarhana, "Security Attacks and Countermeasures for Wireless Sensor Networks: Survey", International Journal of Current Engineering and Technology ISSN 2277 - 4106, June 2013.

[4] Z. BENENSON, M. CHOLEWINSKI, C. FREILING, "Vulnerabilities and Attacks in Wireless Sensor Networks", Laboratory for Dependable Distributed Systems, University of Mannheim, 68131 Mannheim, Germany, 2010

[5] E.Çayırcı and C.Rong ,"Security in Wireless Ad Hoc and Sensor Networks", ISBN: 978-0-47002748-6, 2009.

[6] P. Mohanty, S. Panigrahi, N. Sarma and S. Satapathy, "Security issues in wireless sensor network data gathering protocols: a survey", Department of Computer Science and Engineering Tezpur University, Tezpur, India 2010.

[7] Q. IdreesSarhan, "Security Attacks and Countermeasures for Wireless Sensor Networks: Survey", International Journal of Current Engineering and Technology, 2013.

[8] A.Singla, R. Sachdeva, "Review on Security Issues and Attacks in Wireless Sensor Networks", International Journal of Advanced Research in Computer Science and Software Engineering, Volume 3, Issue 4, April 2013.

[9] V. Soni1, P.Modi, V.Chaudhri, "Detecting Sinkhole Attack in Wireless Sensor Network", International Journal of Application or Innovation in Engineering \& Management, Volume 2, Issue 2, February 2013.

[10] K. Sharma, M.Ghose, "Wireless Sensor Networks: An Overview on its Security Threats", IJCA Special Issue on "Mobile Ad-hoc Networks" MANETs, 2010.

[11] K. Sun, P. Peng, P. Ning, and C. Wang, "Secure Distributed Cluster Formation in Wireless SensorNetworks",in Proceedings of the 22nd AnnualComputer Security Applications Conference (AC-SAC'06), Pages: 131-140, December 2006.

[12] A. Wood and J. Stankovic, "Denial of service insensornetworks",IEEE Computer, pages 5462, October 2002.

[13] Ullah, Fasee, "Analysis of security protocols for Wireless Sensor Networks", Dept. of Comput. Sci., City Univ. of Sci. \& Inf. Technol., Peshawar, Pakistan, Computer Research and Development (ICCRD), 2011. 
International Journal of Wireless \& Mobile Networks (IJWMN) Vol. 5, No. 6, December 2013

[14] A. Perrig, R. Szewczyk, J.D Tygar, V. Wen abd D. Culler, "SPINS:Security Protocols f or Sensor Networks", Departement of electrical engineering and Computer Scinces, University of California, Berkley, USA 2002.

[15] J. Deng, R. Han, S. Mishra, "INSENS: Intrusion-Tolerant Routing in Wireless Sensor Networks", University of Colorado, Department of Computer Science, 2002.

[16] S. Zhu, S. Setia, and S. Jajodia, "LEAP\&plus;Efficient security mechanisms for largescaledistributed sensor networks",ACMTransactionson Sensor Networks (TOSN), Volume 2, Issue 4, November 2006.

[17] S. Saha, Md. Safiqul Islam, Md. SakhawatHossen, "A New OHD Based Intrusion Detection System for Wireless Sensor Network”, IK2206: Internet security and privacy, 2010.

[18] F. Amini, "Simulation and Evaluation of Security and Intrusion detection in IEEE 802.15.4 Network",university of Manitob 2008.

[19] Nabil Ali Alrajeh, S. Khan, and Bilal Shams, "Intrusion Detection Systems in Wireless Sensor Networks: A Review", International Journal of Distributed Sensor Networks, Volume 2013.

[20] Hassen Mohammed AbduallahAlsafi, 2 Saeed Salem Basamh, "A Review of Intrusion Detection System Schemes in Wireless Sensor Network", Journal of Emerging Trends in Computing and Information Sciences, 2013.

[21] Murad A. Rassam, M.A. Maarof and AnazidaZainal, "A Survey of IntrusionDetection Schemes in Wireless Sensor Networks", American Journal of Applied Sciences 9 (10): 1636-1652, 2012.

[22] Andreas A. Strikos, "A full approach for Intrusion Detection in Wireless Sensor Networks", School of Information and Communication Technology, Stockholm, Sweden ,March 1, 2007.

[23] R. Roman, J. Zhou, J. Lopez, "Applying Intrusion Detection Systems to Wireless Sensor Networks", Proceeding of the 3rd IEEE Consumer Communications and Networking Conference, 2006.

[24] Roosta, Tanya, Sameer Pai, Phoebus Chen, Shankar Sastry, and Stephen Wicker. "Inherent security of routing protocols in ad-hoc and sensor networks." In Global TelecommunicationsConference, 2007. GLOBECOM'07. IEEE, pp. 1273-1278. IEEE, 2007.

[25] A. da Silva, M. Martins, B. Rocha, A. Loureiro, L. Ruiz, and H. Wong, "Decentralized intrusion detection in wireless sensor networks", international workshop on Quality of service \& security in wireless and mobile networks, 2005.

[26] C.-C. Su, K.-M.Chang, Y.-H.Kuo, and M.- F. Horng, "The new intrusion prevention and detection approaches for clustering-based sensor networks", in 2005 IEEE Wireless Communications and Networking Conference, WCNC 2005: Broadband Wirelss for the Masses - Ready for Take-off, 2005.

[27] A.Abduvaliyev, A.KPathan, J. Zhou, R. Roman and W. Wong, "On the Vital Areas of Intrusion Detection Systems in Wireless Sensor Networks", Communications Surveys \& Tutorials, IEEE Volume15, Issue 3, 2013.

[28] Mr. Ansar I SheikhMr.PankajKewadkar, "Approach towards Intrusion Detection System for Wireless Sensor Network", International Journal of Advanced Research in Computer and Communication Engineering Vol. 2, 2013.

\section{Authors}

YassineMALEHreceived the B.Sc. degree in networks and Information Systems, from Hassan $1^{\text {st }}$ University, Faculty of Sciences and Technology of Settat, Morocco, in 2009, and M.Sc. degree in Network and Computer engineering from the Hassan $1^{\text {st }}$ University, Faculty of Sciences and Techniques (FSTS), Settat, Morocco, in 2012. Currently pursuing his PhD in Networks and Security Engineeringat the Laboratory of Emerging Technologies (VETE), from Hassan $1^{\text {st }}$ University, Faculty of Sciences and Technology of Settat, Morocco.Hismain research areas arehow to use wireless sensor networks to secure and monitor mobile laboratories networks.

Abdellah EZZATIresearch Scientist in Faculty of Science and Technology in Morocco. He obtained his PHD in 1997 in Faculty of science in Rabat and member of the Computer commission in the same Faculty. Now is an associate professor in Hassan First University in Morocco and he is the Head of Bachelor of Computer Science.He participate to several project as the project Palmes which elaborate a Moroccan Education Certification.

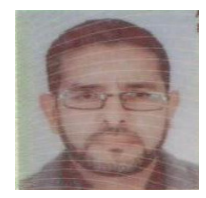

